\title{
Evaluate effects of multiple users in collaborative Brain-Computer Interfaces: A SSVEP study
}

\author{
$1^{\text {st }}$ Tien-Thong Nguyen Do \\ School of Computer Science \\ University of Technology Sydney \\ Ultimo, Australia \\ nguyentienthong.do@uts.edu.au
}

\author{
$2^{\text {nd }}$ Thanh Tung Huynh \\ University of Science and Technology \\ The University of Danang \\ Danang, Vietnam \\ httung@dut.udn.vn
}

\begin{abstract}
This study investigates the effects of collaboration on task performance in brain-computer interface (BCI) based on steady-state visually evoked potential (SSVEP). Navigation tasks were performed in a virtual environment under two conditions, e.g., individual performance and team performance. The results showed that average task completion time in the collaborative condition is decreased by 6 percent compared with that of individual performance, which is inline with other studies in collaborative BCI (cBCI) and joint decision-making. Our work is a step forward for the progress in BCI studies that include multi-user interactions.
\end{abstract}

Index Terms-brain-computer interfaces, SSVEP, CCA, collaborative BCI

\section{INTRODUCTION}

The phenomenon of collective decision-making attracts a lot of interest in the literature recently [1]-[3]. Those researches confirmed that two heads were getting better than one head in the decision-making process, in the case where free communication and interaction are possible between each other. This mechanism is called two-heads-better-than-one (2HBT1) [3]. However, there are still few studies [4]-[6] focusing on the aspect of brain signal decoding. In this study, we investigate this mechanism with a brain-computer interfaces (BCI) system [7]. This work is needed because in a BCI system, typically a game, people tend to play with their teammates. The team performance can be divided into two modes, e.g., collaborative and competitive. In those two modes, users might be encouraged to put more effort, engagement, and attention into the game with their teammates. However, developing such system into the BCI system is still under investigation for the possibility of multiple users in BCI game. Furthermore, the study gives an insight into the impact of multiple users in the cBCI system [8]-[10] compare to individual performances.

One of the first multi-users BCI game reported was Brainball [11]. In this Brainball game, two users have to push a ball one against the other. They are expected to compete by relaxing and their performance is measured by electroencephalography (EEG). The most relaxed player will be the winner. Another study relying on a similar principle can be found in BrainArena [12]. Motor imagery was used to steer a ball into a goal. Eight EEG channels located around the right and left motor cortices were used. This BrainArena game can be played on an individual, collaborative or competitive mode. In

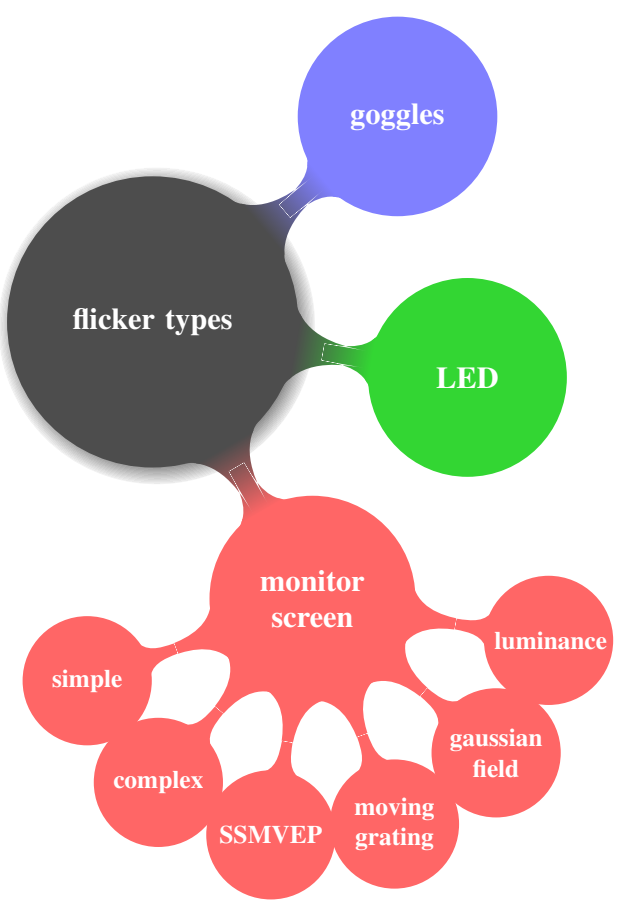

Fig. 1. Flicker types for SSVEP

another collaborative BCI with event-related potentials (ERP) [6], a large number of participants were asked to participate. Six groups of six people were performing the Go/NoGo task in both offline and online conditions. Their results confirmed that collaboration in BCI could accelerate human decisionmaking. So far, only alpha rhythm [11], motor imagery [12] and ERP [6] have been used for online multi-BCIs. There are still open questions regarding other BCI paradigms, such as SSVEP. In Gurkok's study [13], they designed an experiment with multi-players BCI based on SSVEP paradigm, but only one participant was playing the game with EEG control. Participant's partner did not wear an EEG cap, the research target was to check the social impact of collaborative multiBCI game.

In this study, we further investigated the feasibility of multiple users in SSVEP based BCI in cBCI mode in Virtual Environment game. Based on experience with collaborative 
bioRxiv preprint doi: https://doi.org/10.1101/2021.03.05.434173; this version posted March 8, 2021. The copyright holder for this preprint (which was not certified by peer review) is the author/funder, who has granted bioRxiv a license to display the preprint in perpetuity. It is made available under aCC-BY-ND 4.0 International license.

BCI for accelerating human decision-making [14] and other studies with motor imagery and alpha rhythm [11], [12], we sought to imitate the design using a SSVEP paradigm.

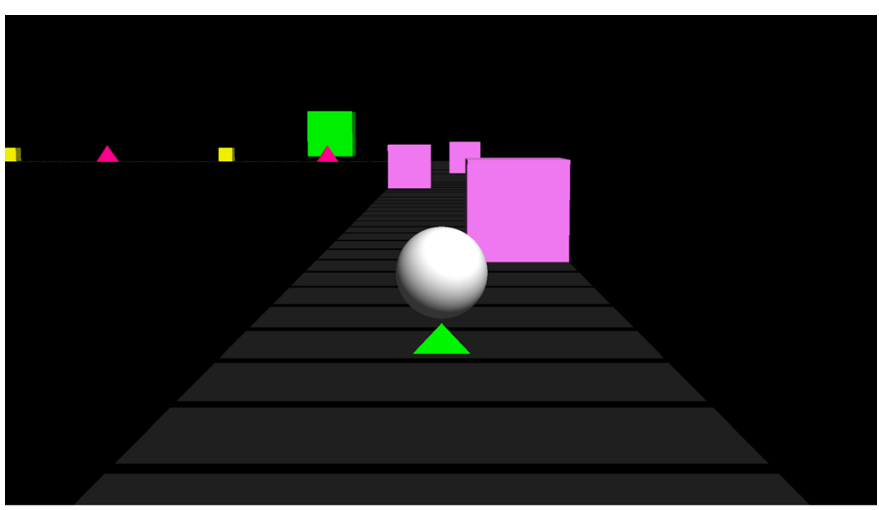

Fig. 2. The experimental user interfaces.

Collaborative SSVEP BCI experimental procedure

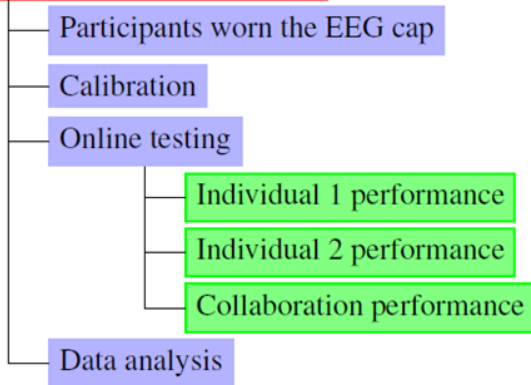

Fig. 3. Experimental procedure.

\section{METHOD}

\section{A. Participants}

Ten graduate students ( 5 females: mean age $25.3 \pm 0.8$ years old ranging from 24 and 26) voluntarily participated in this experiment. They all had normal or corrected to normal vision. No psychiatric disorders were reported from themselves and their family members. Members of each pair knew each other.

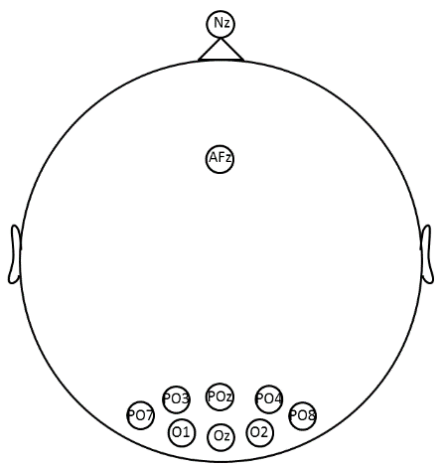

Fig. 4. The EEG electrode channels were used in this experiment.

\section{B. Experimental Design}

SSVEP technique SSVEP is a synchronized brain response induced by a repetitive visual stimulus, flickering at a constant frequency [15]. The principle is same for different kinds of stimuli types, oscillating or moving image with constant frequency (stimulus frequency) will elicit brain response to the same stimulus frequency and its harmonics.

There are several stimuli types with different level of complexity ranging from simple to complex (figure 1) [15]

- goggles [16]

- light-emitting diodes (LED) [17]

- monitor screen: liquid crystal display (LCD) or cathode ray tube (CRT)

- simple: just black-white color

- complex pattern: checkerboard [18], emotional pictures [19]

- steady-state motion visual evoked potentials [20]

- moving grating

- gaussian field [21]

- intensity of the monitor luminance [22]

a
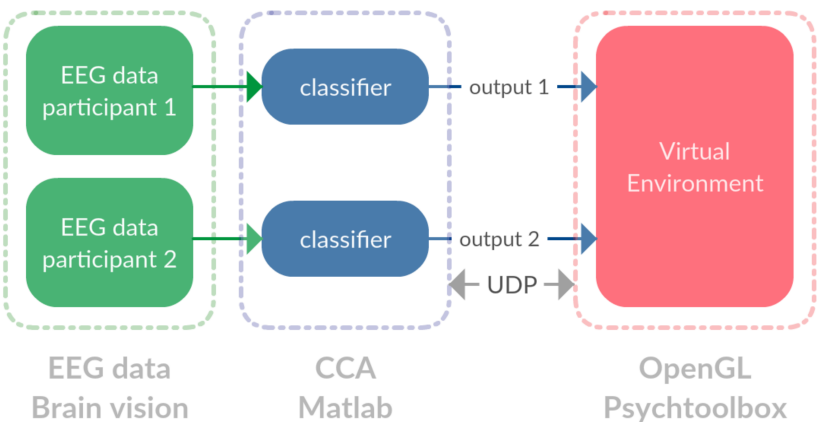

OpenGL

Brain vision Matlab

Psychtoolbox

Matlab

b

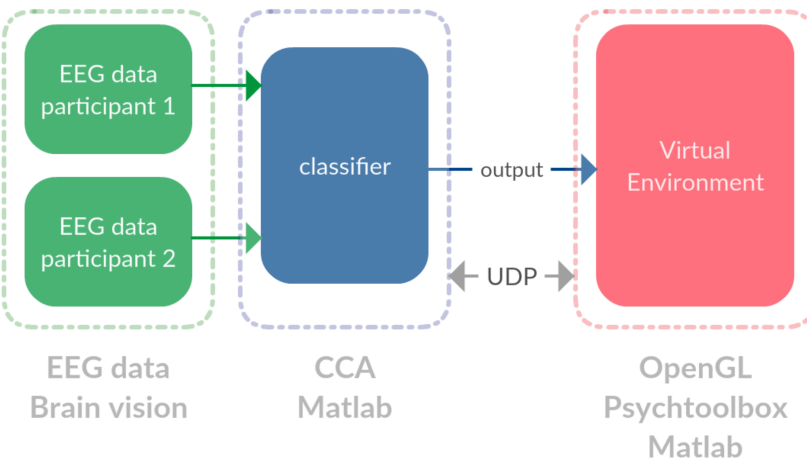

Fig. 5. Collaborative scheme setup for (a) type 1 and (b) type 2, respectively.

In this study, we chose the intensity of the monitor luminance to generate flicker. This method can be adapted and incorporated into the monitor, which can be more flexible for user feedback. The user interface was created with OpenGL embedded in Psychophysics Toolbox-3 for MATLAB [23] (figure 2). Through this user interface, the participant can control the ball's movement with three commands: forward, left, and right. This game's mission is to move forward the ball while avoiding obstacles and reach the destination as 
fast as possible. The completion time was measured as user performance.

\section{Experimental procedure}

Figure 3 describes the procedure of our experiment. In the collaborative BCI task, five dyads of participants (two participants $\mathrm{x}$ five teams) wore EEG caps, and their data were recorded simultaneously. Participants sat in a comfortable chair with armrests and were given instructions regarding the experimental procedure. A 32 multi-channel EEG amplifier (BrainAmp by Brain Products, Munich, Germany) was used to record the EEG signal. The data were recorded at 1000 $\mathrm{Hz}$ with a band-pass filter of 0.5-50 Hz. Eight EEG channels were recorded in the occipital area: PO3, PO4, PO7, PO8, $\mathrm{POz}, \mathrm{O} 1, \mathrm{O} 2$, and $\mathrm{Oz}$, according to the international 10-20 system (Figure 4). The nose and $\mathrm{AFz}$ were used as reference and ground, respectively. The impedance was kept below 10 $\mathrm{k} \Omega$ throughout the experiment.

Participants were instructed to pay attention to a square filled with a high arousal picture in the calibration session. The emotional picture continuously oscillated at a frequency of $6 \mathrm{~Hz}, 7.5 \mathrm{~Hz}$, and $10 \mathrm{~Hz}$, respectively. Twenty trials were recorded for each case $(20$ trials for the $6 \mathrm{~Hz}, 20$ trials for the $7.5 \mathrm{~Hz}$, and 20 trials for the $10 \mathrm{~Hz}$ condition), and each trial lasted for $12 \mathrm{~s}$. To minimize carryover effects, an interval of $4 \mathrm{~s}$ was used between trials, and a resting time of $10 \mathrm{~s}$ was allocated between each frequency condition. It took about 15 minutes for each participant to complete the calibration session. Following the calibration session, optimal parameters were chosen by the canonical correlation analysis (CCA) algorithm.

Figure 5 shows the schematic design for this experiment. Generally, the frameworks of both schemes are same and have three main steps:

- Brain signal acquisition from Brain vision hardware.

- CCA algorithm for classifying user intention. The output of the classifier was used as the input for virtual environment game. Both virtual environment and classifier communicate with each other by user datagram protocol (UDP).

- Virtual environment plays the role of visual feedback.

In addition, two different strategies are considered for analyzing SSVEP signal of multiple players:

- Multiple input commands for Virtual Environment (figure 5a ). The EEG data of each participant was classified separately to give out two independent commands to control the ball movement in the virtual environment.

- Single input command for the Virtual Environment (figure $5 b$ ). Both EEG user data from Brain vision hardware were merged together and classified by CCA to give out the single input for virtual environment.

\section{Data processing}

In this study, we used CCA method for the classification [24], [25]. CCA is a machine learning method that enables to

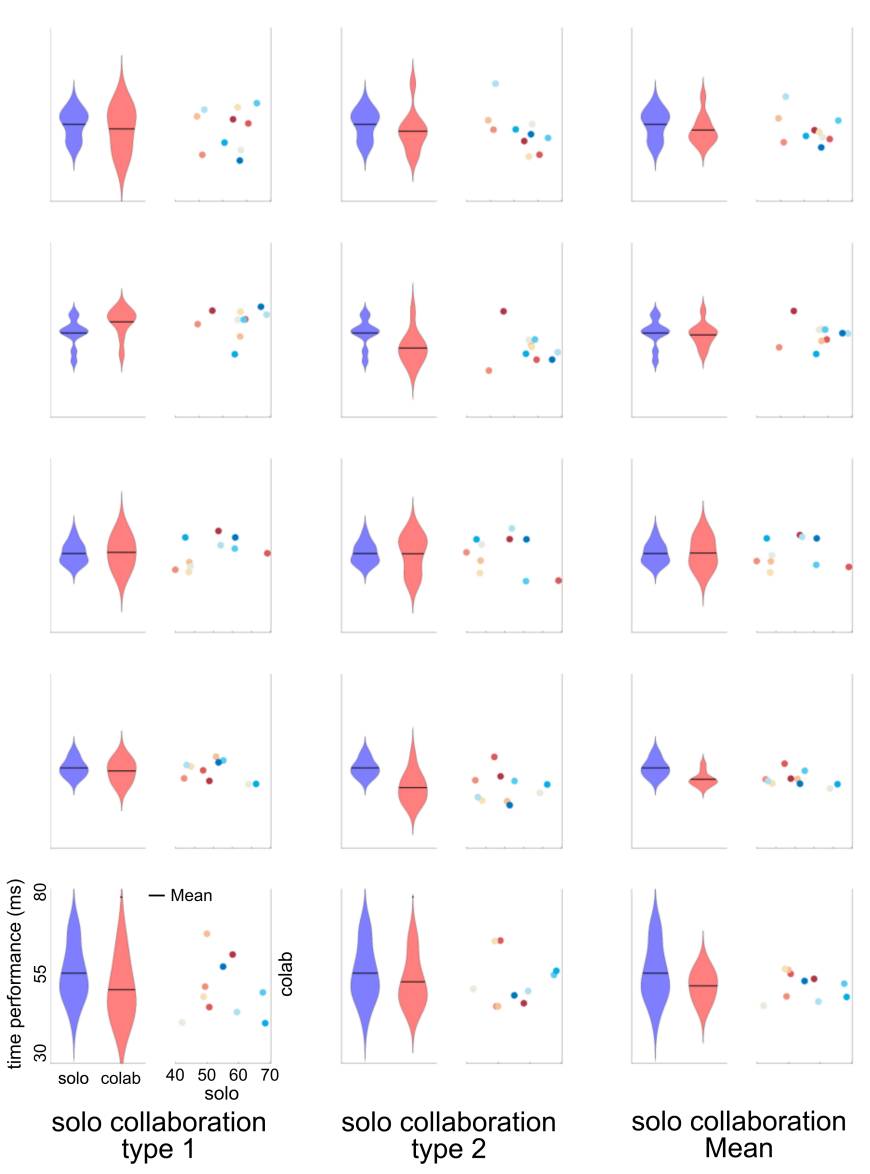

Fig. 6. Time performance for each pair.

find an underlying correlation between two sets of variables [26]. For two sets of variables $\mathrm{X}$ and $\mathrm{Y}, \mathrm{CCA}$ method consists of finding vectors $\mathrm{W}$ and $\mathrm{v}$ that maximize the correlation between $w^{T} \mathrm{X}$ and $v^{T} \mathrm{Y}$.

$\rho(x, y)=\max _{w, v} \frac{E\left[\widetilde{x} \widetilde{y}^{T}\right]}{\sqrt{E\left[\widetilde{x} \widetilde{x}^{T}\right] E\left[\widetilde{y} \widetilde{y}^{T}\right]}}=\max _{w, v} \frac{w^{T} X Y v}{\sqrt{w^{T} X X^{T} w v^{T} Y Y^{T} v}}$

where $\rho$ indicates the maximum correlation coefficient, $\mathrm{X}$ refers to the set of multi-channel EEG data signals, and $\mathrm{Y}$ is reference signals set that have the same length as $\mathrm{X}$ :

$$
Y_{i}=\left(\begin{array}{c}
\cos \left(2 \pi f_{i} t\right) \\
\sin \left(2 \pi f_{i} t\right) \\
\vdots \\
\cos \left(2 \pi H f_{i} t\right) \\
\sin \left(2 \pi H f_{i} t\right)
\end{array}\right), \quad t=\frac{1}{F s}, \ldots, \frac{S}{F s}
$$

$\mathrm{H}$ is the number of harmonics, the classifier's output is recognized as:

$$
f_{\text {target }}=\max _{i} \rho_{i}, \quad i=1,2, \ldots, K
$$




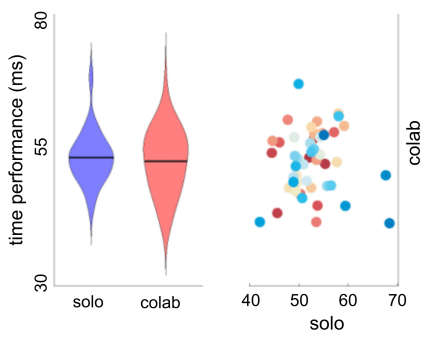

Average performance of type 1
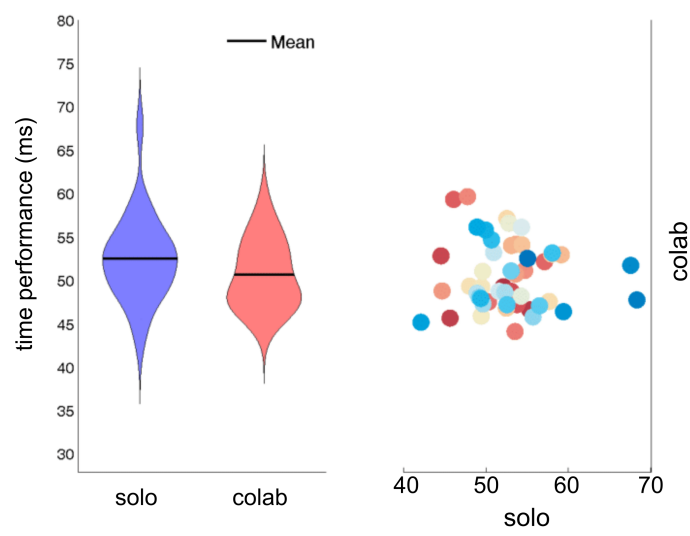

Average performance of solo and collaboration

Fig. 7. The average of time performance.

\section{RESULTS}

\section{A. Behavioral performance}

As shown in the figure 6, figure 7, participants showed better performance in the collaboration condition compared to those in the individual conditions. The collaboration results tended to show a reduced completion time of up to 6 percent. While earlier studies have reported increased performances for collaboration-based multiuser BCIs in ERP-based [6], or MI-based paradigms [12], here we demonstrate the evidence of a positive effect of synchronizing collaboration for online SSVEP-based BCI.

\section{B. EEG data analysis}

The more channels we used for classifying data under the machine learning aspect, the more accuracy we might get. This trend can be seen clearly in the figure 8 , and figure 9 . On the other hand, under the enjoyment aspect illustrated in figure 7 , we could see that when people performed collaborative task, they tend to be more involved than individual performance.

\section{Discussion AND CONCLUSIONS}

Our work showed that multiple users could benefit from a synergetic effect of collaboration compared with individual performance in terms of brain signal decoding. It also provides another solution for end-users interaction with the BCI system. However, the present work lacks a comparison of a larger majority of machine learning techniques for classifying user

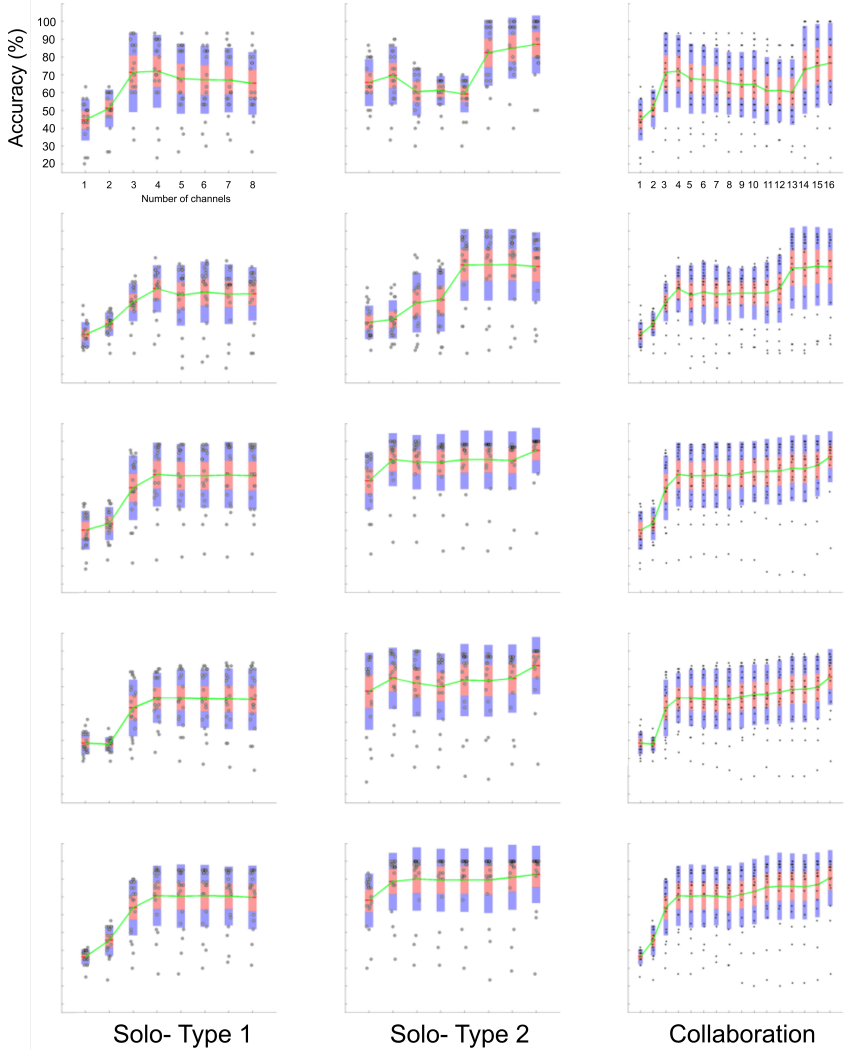

Fig. 8. Classifier accuracy of each pair.

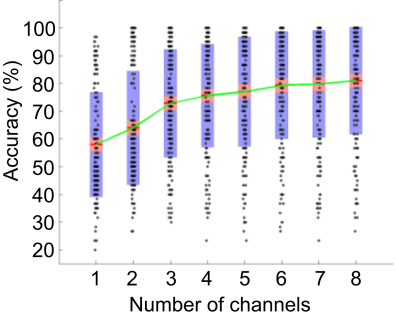

Average classifier accuracy by using different channel number in solo setup

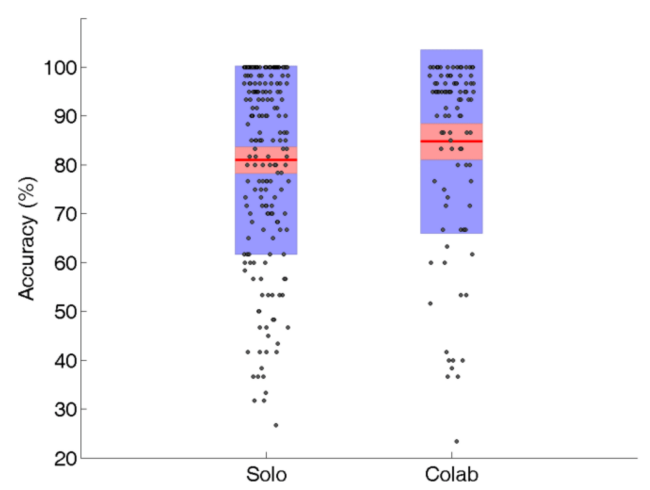

Average classifier accuracy in solo and collaboration

Fig. 9. The average of classifier accuracy. 
intention that should be considered for future works. Another factor that needs to be considered is system response time, as it is a trade-off with the classifier accuracy. Finally, the adaptive user-dependant factor could be examined based on the confidence levels and flicker frequency. For instance, a participant with higher confidence can have a higher weight factor in the classifier.

\section{REFERENCES}

[1] B. Bahrami, K. Olsen, P. E. Latham, A. Roepstorff, G. Rees, and C. D. Frith, "Optimally interacting minds," Science, vol. 329, no. 5995, pp. 1081-1085, 2010.

[2] M. O. Ernst, "Decisions made better," Science, vol. 329, no. 5995, pp. 1022-1023, 2010.

[3] A. Koriat, "When are two heads better than one and why?" Science, vol. 336, no. 6079, pp. 360-362, 2012.

[4] M. P. Eckstein, K. Das, B. T. Pham, M. F. Peterson, C. K. Abbey, J. L. Sy, and B. Giesbrecht, "Neural decoding of collective wisdom with multi-brain computing," NeuroImage, vol. 59, no. 1, pp. 94-108, 2012.

[5] A. Stoica, "Multimind: Multi-brain signal fusion to exceed the power of a single brain," 2012 Third International Conference on Emerging Security Technologies, pp. 94-98, 2012.

[6] P. Yuan, Y. Wang, X. Gao, T.-P. Jung, and S. Gao, "A collaborative braincomputer interface for accelerating human decision making," International Conference on Universal Access in Human-Computer Interaction, pp. 672-681, 2013.

[7] C.-T. Lin and T.-T. N. Do, "Direct-sense brain-computer interfaces and wearable computers," IEEE Transactions on Systems, Man, and Cybernetics: Systems, vol. 51, no. 1, pp. 298-312, 2021.

[8] D. Valeriani, C. Cinel, and R. Poli, "Hybrid collaborative braincomputer interfaces to augment group decision-making," Neuroergonomics, pp. 187-190, 2019.

[9] D. Valeriani, R. Poli, and C. Cinel, "Enhancement of group perception via a collaborative brain-computer interface," IEEE Transactions on Biomedical Engineering, vol. 64, no. 6, pp. 1238-1248, 2016.

[10] D. Valeriani, C. Cinel, and R. Poli, "Group augmentation in realistic visual-search decisions via a hybrid brain-computer interface," Scientific reports, vol. 7, no. 1, pp. 1-12, 2017.

[11] S. I. Hjelm and C. Browall, "Brainball-using brain activity for cool competition," in Proceedings of NordiCHI, vol. 7, no. 9, 2000.

[19] A. Keil, T. Gruber, M. M. Müller, S. Moratti, M. Stolarova, M. M. Bradley, and P. J. Lang, "Early modulation of visual perception by emotional arousal: evidence from steady-state visual evoked brain potentials," Cognitive, Affective, \& Behavioral Neuroscience, vol. 3, no. 3, pp. 195-206, 2003.
[12] L. Bonnet, F. Lotte, and A. Lécuyer, "Two brains, one game: design and evaluation of a multiuser BCI video game based on motor imagery," IEEE Transactions on Computational Intelligence and AI in games, vol. 5, no. 2, pp. 185-198, 2013.

[13] H. Gürkök, A. Nijholt, M. Poel, and M. Obbink, "Evaluating a multiplayer brain-computer interface game: Challenge versus co-experience," Entertainment Computing, vol. 4, no. 3, pp. 195-203, 2013.

[14] Y. Wang and T.-P. Jung, "A collaborative brain-computer interface for improving human performance," PloS one, vol. 6, no. 5, p. e20422, 2011.

[15] F.-B. Vialatte, M. Maurice, J. Dauwels, and A. Cichocki, "Steady-state visually evoked potentials: focus on essential paradigms and future perspectives," Progress in neurobiology, vol. 90, no. 4, pp. 418-438, 2010.

[16] S. P. Koch, J. Steinbrink, A. Villringer, and H. Obrig, "Synchronization between background activity and visually evoked potential is not mirrored by focal hyperoxygenation: implications for the interpretation of vascular brain imaging," The Journal of neuroscience, vol. 26, no. 18, pp. 4940-4948, 2006.

[17] T. Sakurada, T. Kawase, T. Komatsu, and K. Kansaku, "Use of highfrequency visual stimuli above the critical flicker frequency in a SSVEPbased BMI," Clinical Neurophysiology, 2014.

[18] H. Bakardjian, T. Tanaka, and A. Cichocki, "Optimization of SSVEP brain responses with application to eight-command Brain-Computer Interface," Neuroscience letters, vol. 469, no. 1, pp. 34-38, 2010.

[20] J. Xie, G. Xu, J. Wang, F. Zhang, and Y. Zhang, "Steady-state motion visual evoked potentials produced by oscillating newton's rings: Implications for brain-computer interfaces," PloS one, vol. 7, no. 6, p. e39707, 2012.

[21] J. Xie, G. Xu, J. Wang, S. Zhang, F. Zhang, Y. Li, C. Han, and L. Li, "Addition of visual noise boosts evoked potential-based brain-computer interface," Scientific reports, vol. 4, 2014.

[22] X. Chen, Z. Chen, S. Gao, and X. Gao, "A high-ITR SSVEP-based BCI speller," Brain-Computer Interfaces, vol. 1, no. 3-4, pp. 181-191, 2014.

[23] D. H. Brainard, "The psychophysics toolbox," Spatial vision, vol. 10, no. 4, pp. 433-436, 1997.

[24] G. Bin, X. Gao, Z. Yan, B. Hong, and S. Gao, "An online multi-channel SSVEP-based brain-computer interface using a canonical correlation analysis method," Journal of neural engineering, vol. 6, no. 4, p. 046002, 2009.

[25] X. Chen, Y. Wang, S. Gao, T.-P. Jung, and X. Gao, "Filter bank canonical correlation analysis for implementing a high-speed SSVEP-based braincomputer interface," Journal of neural engineering, vol. 12, no. 4, p. 046008, 2015.

[26] H. Hotelling, "Relations between two sets of variates," Breakthroughs in statistics, pp. 162-190, 1992. 